\title{
Estrategia colaborativa basada en la reactancia de falla y la firma del sistema para la localización de fallas en sistemas de distribución
}

ELECTRICAL ENGINEERING

\section{Collaborative strategy based on the fault reactance and the system signature for locating faults in power distribution systems}

\author{
H. Peláez-Álzate*, J. Mora-Flórez ${ }^{{ }^{*}}$, S. Pérez-Londoño* \\ * Programa de Ingeniería Eléctrica, Universidad Tecnológica de Pereira, Pereira, Colombia \\ hjpelaez@utp.edu.co,sjjmora@utp.edu.co,saperez@utp.edu.co
}

(Recibido: Marzo 10 de 2011 - Aceptado: Octubre 25 de 2011)

\begin{abstract}
Resumen
En este artículo se presenta una estrategia colaborativa para la localización de fallas en sistemas de distribución, basada en el análisis gráfico de la impedancia de falla y en la firma del sistema, a partir de las medidas de tensión y corriente en solo un terminal de la línea. Esta estrategia permite resolver el problema de la múltiple estimación del nodo en falla, que se presenta debido a la topología altamente ramificada, propia de los sistemas de distribución. Según las pruebas realizadas en un sistema de distribución prototipo, considerando fallas monofásicas, bifásicas y trifásicas, y resistencias de falla que varían de $0 \mathrm{a}$ $40 \Omega$, se obtuvieron errores absolutos promedio en la localización del 5.2\% aproximadamente. Se aprecia por tanto, que la estrategia propuesta resulta muy eficiente para la localización de fallas en sistemas de distribución.
\end{abstract}

Palabras Clave: Localización de fallas, Sistemas de distribución, Múltiple estimación de fallas.

\begin{abstract}
A collaborative strategy for fault location in power distribution systems based on the fault impedance and the system signature, and using only single-end measurements, is presented in this paper. This strategy is useful to solve the multiple estimation of the faulted node, which is due to the presence of several laterals in the power system topology. According to the tests performed in a prototype power distribution system, considering single phase faults, phase to phase faults and three phase faults, and also using fault resistances from 0 to $40 \Omega$, the obtained results show an average absolute error of 5,2\% approximately. From the results can be concluded that this proposal is efficient to locate faults in power distribution systems.
\end{abstract}

Keywords: Fault location, Power distribution systems, Multiple estimation of the fault location. 


\section{Introducción}

Debido al crecimiento acelerado de los sistemas eléctricos de potencia, a la incursión de nuevas tecnologías de automatización y a las exigencias de los entes reguladores asociadas con la calidad del servicio, en la última década se ha brindado mayor atención a los sistemas de distribución (CREG, 2008; T. A. Short, 2007; Philipson \& Willis, 2005).

Uno de los problemas que afectan la calidad y está asociado con la continuidad del suministro, es la presencia de fallas en la red. En este campo se han realizado múltiples investigaciones para disminuir los tiempos de interrupción del servicio de energía eléctrica (Mora, 2006; Zhu et al., 1997; Das, 1998). En sistemas de transmisión de energía eléctrica, el problema de la localización de fallas es más fácil de solucionar, debido a que en éstos se dispone de más información como las medidas de tensión y corriente en ambos terminales de las líneas, las cuales además no tienen derivaciones, son transpuestas y de configuración homogénea. A diferencia de los sistemas de transmisión, la localización de fallas en los sistemas de distribución presenta un grado de complejidad más alto, debido a que no son sistemas balanceados, tienen topología radial con muchas ramificaciones y tienen múltiples calibres de conductores. La alta ramificación de la red es la causante del problema de la múltiple estimación de la falla, debido que un algoritmo de localización basado en medidas en un solo terminal de la línea, localiza varios nodos normalmente ubicados en diferentes ramales, donde se estima la localización de la falla (Mora et al., 2009).

Existen muchos métodos que calculan la distancia a la falla, pero solo algunos de ellos proponen una solución al problema de la múltiple estimación (Morales et al., 2009). Otras estrategias de localización, se fundamentan en los métodos basados en la extracción del conocimiento útil de las bases de datos de registros de fallas; sin embargo, tienen el inconveniente de su alto costo computacional y su dificultad para el ajuste (Butler \& Momoh, 2000; Gutierrez-Gallego et al., 2010; Hagh et al., 2007).
Como consecuencia de lo expuesto, es necesario desarrollar un método complementario e integrado, que además de estimar un punto de falla de una manera confiable, reduzca el problema de la múltiple estimación mediante la identificación del ramal en falla, con un bajo costo computacional y fácil ajuste.

Este artículo presenta un método cooperativo para localizar fallas en sistemas de distribución, a partir de dos estrategias: la primera es el análisis del gráfico de reactancias basada en lo propuesto en (Morales et al., 2010), la cual determina la distancia de falla medida desde la subestación al punto de falla, a partir de la impedancia medida en un extremo de la línea. La segunda estrategia permite determinar la zona fallada, mediante el análisis del comportamiento de las corrientes del sistema bajo falla y su relación con las protecciones del sistema (Seung-Jae L. et al. 2004). Esta última estrategia se fundamenta entonces en la utilización de la señales de corriente, que en este artículo se denomina como la firma del sistema. Mediante la combinación sencilla de estas dos estrategias, se permite una aplicación inmediata para los operadores de red, con una precisión aceptable y con un bajo costo computacional.

El artículo se presenta en cinco secciones. En la sección dos se presentan los aspectos básicos de las estrategias utilizadas para localización de fallas. En el capítulo tres se presenta una propuesta para la integración colaborativa de los métodos de localización basados en la firma del sistema y el gráfico de reactancias. En el capítulo cuatro se realizan pruebas y se analizan los resultados para un sistema de distribución prototipo. Finalmente, en el capítulo cinco se presentan las conclusiones más relevantes de la investigación desarrollada.

\section{Aspectos básicos de las estrategias utilizadas para localización de fallas}

El problema de la múltiple estimación de la localización de fallas en circuitos de distribución, se puede resolver mediante la implementación de algoritmos que utilizan el modelo de la red y los que aprovechan otro tipo de información del sistema. Los fundamentos teóricos básicos de la 
propuesta que integra el método basado en la estimación de la reactancia de falla y la firma del sistema, se presentan en esta sección.

\subsection{Método basado en el análisis de la firma del sistema}

El método basado en la firma del sistema utiliza el análisis de las señales de corriente registradas en la subestación y aprovecha las características de operación de las protecciones del sistema, para identificar la zona de ocurrencia de las fallas (Seung-Jae L. et al. 2004). Para aplicar este método se requiere que el circuito tenga instalado elementos de protección o automatización, tales como reconectadores, seccionalizadores y fusibles, a lo largo de sus alimentadores.

A manera de ejemplo, en el nodo 19 del circuito de prueba de la Figura 1, se simula una falla monofásica $\mathrm{B}$ a tierra, con resistencia de falla de $0,5 \Omega$. Las señales de corriente medidas en la subestación se presentan en la Figura 2.
De los registros de corriente tomados en la subestación de distribución, en Figura 2 se observa que para la señal de la fase $\mathrm{B}$, se presenta un incremento significativo en la magnitud de la corriente. Este incremento se presenta en los intervalos de tiempo A, C, E y G, y se caracteriza por poseer una magnitud de corriente alta y una duración equivalente al tiempo de despeje de la protección activa. En el intervalo marcado con P se encuentra el estado de prefalla. En los intervalos $\mathrm{B}, \mathrm{D}$ y $\mathrm{F}$ se ha despejado la falla por parte del reconectador $\mathrm{RC} 1$, a través de su curva rápida. En el intervalo $\mathrm{H}$ se despeja la falla totalmente por acción del fusible F1, cuando el reconectador se prepara para operar con su curva lenta. La duración de los intervalos B, D y F, está dada por el tiempo de re-cierre programado en el relé o reconectador actuante. Este modo de operar de las protecciones del sistema es lo que se conoce como "salvamento de fusibles", muy utilizado en sistemas de distribución (Phadke\&Horowitz, 1995; T. A. Short, 2007).

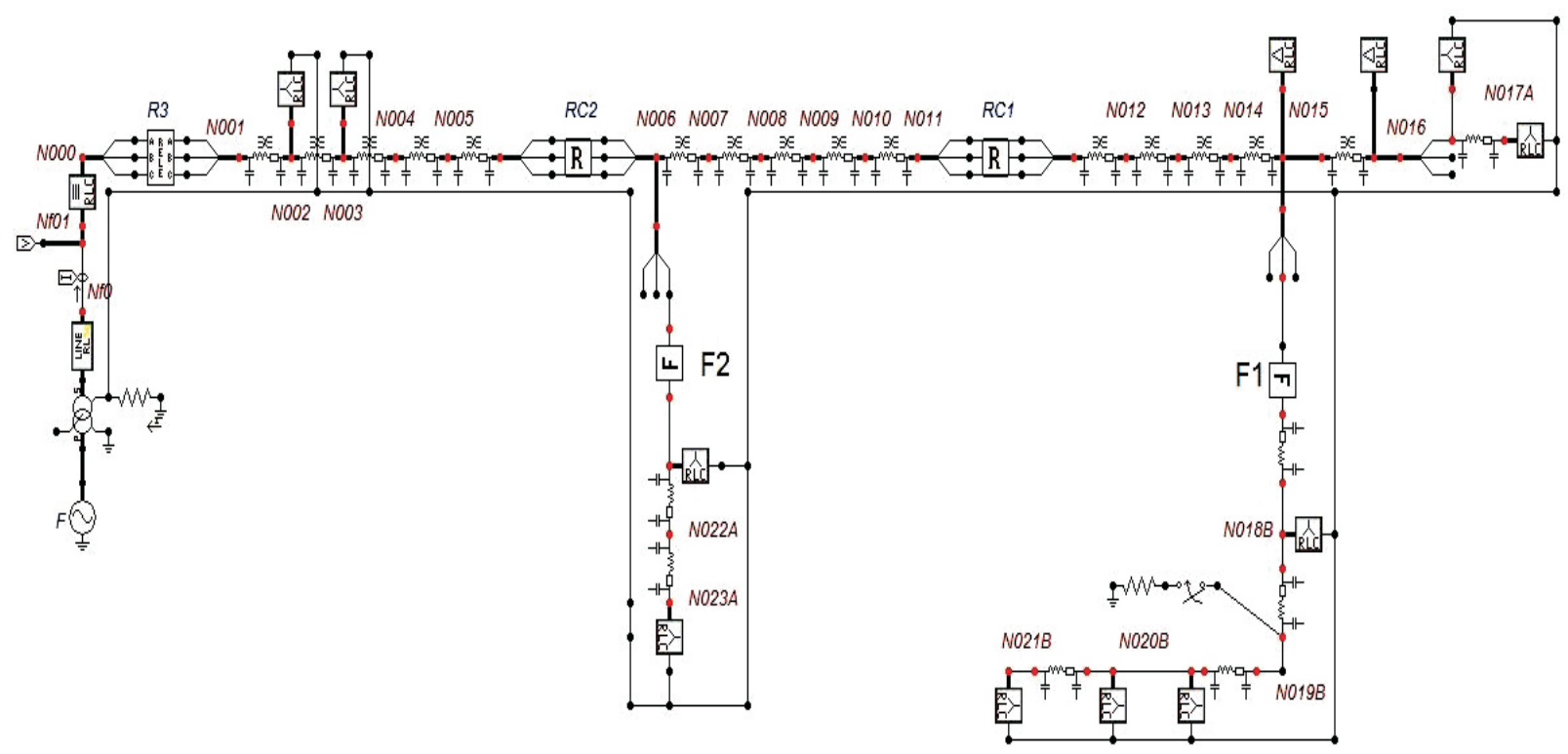

Figura 1. Falla monofásica B-tierra, en el nodo 19 del sistema de prueba 


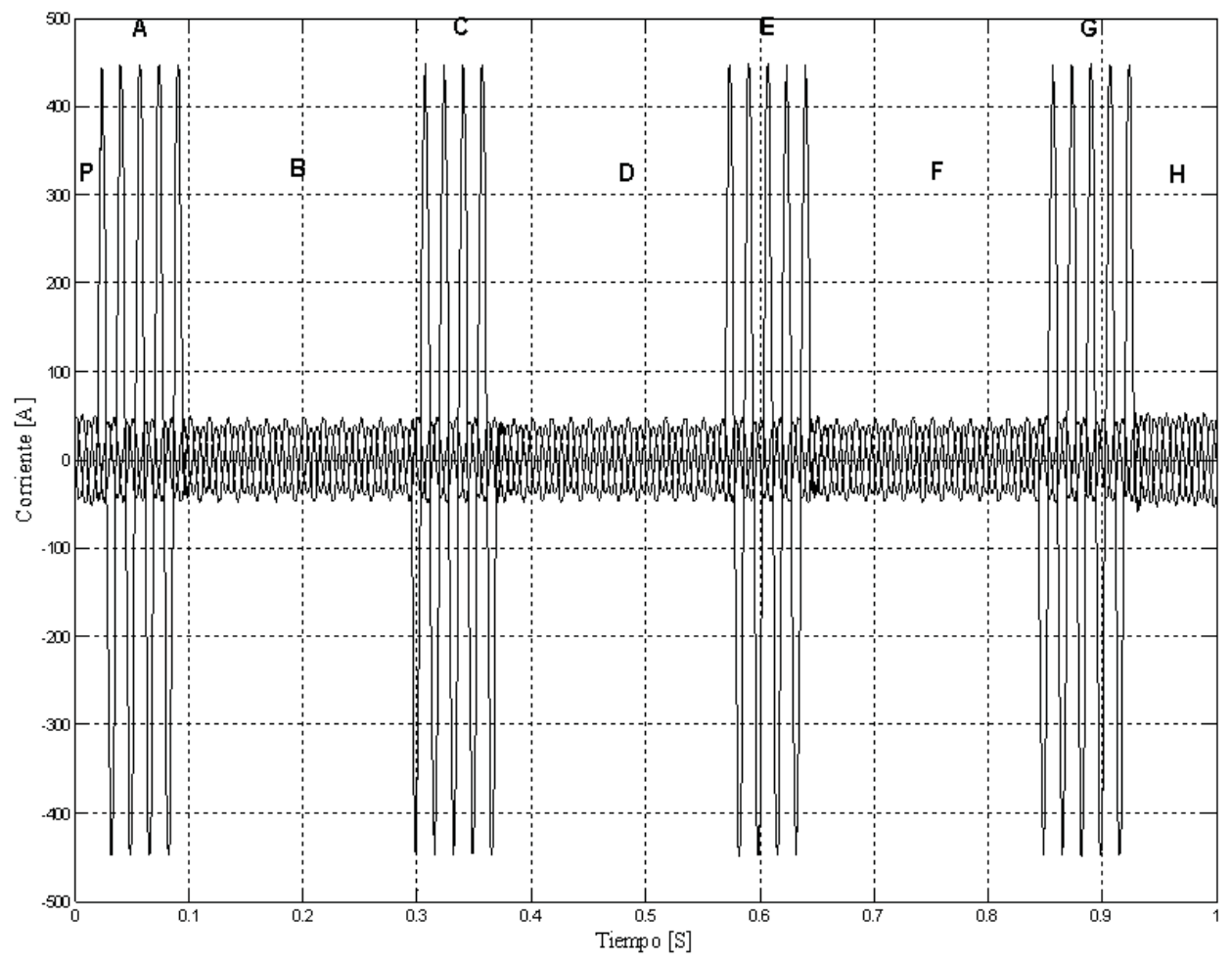

Figura 2. Señales de corriente registradas en la subestación, ante una falla B a tierra en el nodo 19 del sistema de prueba

Cada dispositivo del sistema de protección tiene sus características particulares, por ejemplo el reconectador RC1 está programado para efectuar tres operaciones de apertura por curva rápida y una por curva lenta; además, efectúa los re-cierres cada 0,2 segundos, el cual es el tiempo de duración de los intervalos de tiempo B, D y F de la Figura 2. Otra característica útil para propósitos de la localización de fallas es la relación entre la corriente de falla y el tiempo de retardo de la apertura, según los ajustes realizados en la coordinación (Std. IEEE C37.112, 1996). Para $\mathrm{RC} 1$ este tiempo de retardo en la apertura se observa en los intervalos A, C y E. La firma del sistema hace referencia a esa serie de características asociadas a la operación de las protecciones del sistema, que se registran en las señales de corriente tomadas en la subestación (Mora, 2006). Para encontrar la zona donde ocurre una falla en un sistema de distribución basta con identificar los tiempos de operación (despeje y re- cierre) de las protecciones que actuaron al despejarla. Para esto, es necesario conocer plenamente la ubicación de todos los dispositivos de protección en el circuito y sus características de operación. En el caso del ejemplo, se detecta que los dispositivos que actuaron para despejar la falla fueron RC1 y F1, la zona de falla se ubica entonces aguas abajo de estos dos dispositivos. Para que la estrategia funcione, se debe conocer la configuración de cada protección, y adicionalmente, los tiempos de re-cierre para cada reconectador pueden ser sutilmente diferentes en algunos milisegundos, sin que esto afecte su funcionalidad en el sistema de potencia.

\subsection{Método de estimación de la distancia de falla basado en el análisis del gráfico de reactancia}

Este método se basa en la determinación de la reactancia de falla $X_{F}$ de un circuito, en función de 
una distancia de falla variable $m$. Se asume que la distancia de falla es la correcta, cuando la reactancia de falla de la fase o las fases falladas alcanza su valor mínimo, partiendo de la concepción generalizada de que la impedancia de falla es puramente resistiva. La técnica se desarrolla considerando las restricciones propias de los sistemas de distribución como son la monitorización de tensión y corriente en un terminal de la línea (subestación), desbalance del sistema, variación de la carga, redes no homogéneas y efecto de la resistencia de falla (Morales et al., 2010).

Para el desarrollo del método se utilizan los fasores de tensión y corriente de prefalla y falla en la subestación, además de la impedancia serie de la línea de cada tramo del circuito. Adicionalmente, la carga se concentra al final del alimentador, ya que no es posible establecer el valor de la carga en cada momento, tomando únicamente medidas en la subestación. El error cometido con esta aproximación tiende a ser pequeño, dado que en la mayoría de sistemas de distribución la impedancia de carga $\boldsymbol{Z}_{q}$ es mucho mayor a la impedancia de línea $\boldsymbol{Z}_{L}$.

\section{i. Análisis para condiciones de prefalla}

En la Figura 3 se presenta la simplificación del sistema propuesto para el análisis en condiciones de prefalla. $\boldsymbol{Z}_{L}$ es la matriz de impedancia serie de la línea, $\boldsymbol{Z}_{q}$ es la matriz de impedancia de carga y $\boldsymbol{V}_{\text {abc }}^{\mathrm{p}}$ y $\boldsymbol{I}_{\text {abcp }}^{\mathrm{p}}$ son los vectores que contienen los fasores de tensión y corriente de prefalla por fase, medidos en la subestación. Estas variables tienen la forma que se presenta en Ec. (1), (2), (3) y (4), respectivamente.

\section{$\mathrm{S} / \mathrm{E}$}

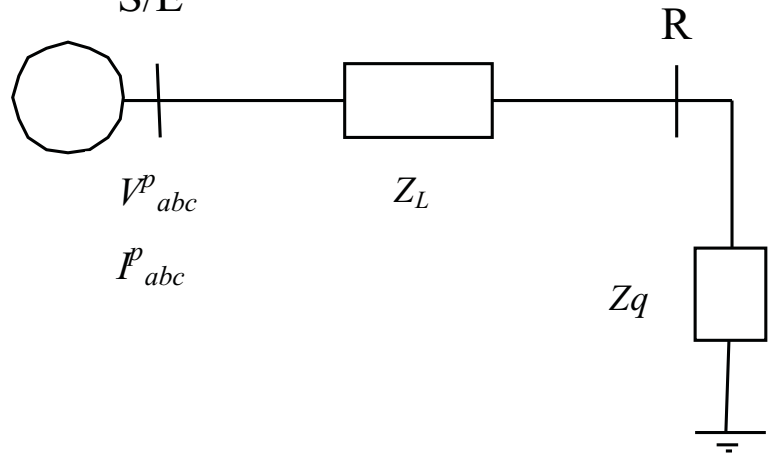

Figura 3. Circuito simplificado de un sistema de distribución antes de la falla.

$$
\begin{gathered}
\boldsymbol{L}=\left[\begin{array}{lll}
Z_{a a} & Z_{a b} & Z_{a c} \\
Z_{b a} & Z_{b b} & Z_{b c} \\
Z_{c a} & Z_{c b} & Z_{c c}
\end{array}\right] \\
\boldsymbol{Z}_{\boldsymbol{q}}=\left[\begin{array}{ccc}
Z_{q a} & 0 & 0 \\
0 & Z_{q b} & 0 \\
0 & 0 & Z_{q c}
\end{array}\right] \\
\boldsymbol{V}_{a b c}^{p}=\left[\begin{array}{c}
V_{a p} \\
V_{b p} \\
V_{c p}
\end{array}\right] \\
\boldsymbol{I}_{a b c}^{p}=\left[\begin{array}{l}
I_{a p} \\
I_{b p} \\
I_{c p}
\end{array}\right]
\end{gathered}
$$

La matriz de impedancia de carga $\boldsymbol{Z}_{\mathrm{q}}$ es del tipo impedancia constante y en conexión $\mathrm{Y}$, y se determina a partir de las condiciones de prefalla, considerando un sistema con neutro. De la Figura 3 se obtiene Ec.(5).

$$
\boldsymbol{V}_{a b c}^{p}=\left(\boldsymbol{Z}_{\boldsymbol{L}}+\boldsymbol{Z}_{\boldsymbol{q}}\right) \times \boldsymbol{I}_{a b c}^{p}
$$

Al despejar $\boldsymbol{Z}_{\mathrm{q}}$ de la Ec. (5) se obtiene Ec.(6) y el conjunto de Ec.(7).

$$
\left(Z_{q} \times I_{a b c}^{p}\right)=V_{a b c}^{p}-\left(Z_{L} \times I_{a b c}^{p}\right)
$$

$$
\begin{aligned}
& Z_{q a}=\frac{V_{a p}}{I_{a p}}-\frac{Z_{a a} \times I_{a p}+Z_{a b} \times I_{b p}+Z_{a c} \times I_{c p}}{I_{a p}} \\
& Z_{q b}=\frac{V_{b p}}{I_{b p}}-\frac{Z_{b a} \times I_{a p}+Z_{b b} \times I_{b p}+Z_{b c} \times I_{c p}}{I_{b p}} \\
& Z_{q c}=\frac{V_{c p}}{I_{c p}}-\frac{Z_{c a} \times I_{a p}+Z_{c b} \times I_{b p}+Z_{c c} \times I_{c p}}{I_{c p}}
\end{aligned}
$$


La carga se concentra al final del circuito para efectos prácticos, como se mencionó anteriormente, pero el método permite acumular las cargas de diversas maneras, cuando se conoce la característica de distribución a partir de medidas locales en cada lateral de carga.

\subsection{Análisis para condiciones de falla.}

El circuito que se propone para condiciones de falla se presenta en la Figura 4, donde $\boldsymbol{Z}_{F}$ representa la impedancia de falla, $m$ es la distancia de falla en $p . u$ medida desde la subestación y $\boldsymbol{V}_{a b c}^{f}$ y $\boldsymbol{I}_{a b c}^{f}$ son los vectores de tensión y corriente de falla por fase, los cuales conservan la estructura $\operatorname{de} \boldsymbol{V}_{a b c p}^{p}$ y $\boldsymbol{I}_{a b c}^{p}$. De la Figura 4, se pueden obtener (8), (9) y (10) para la tensión, corriente e impedancia en el nodo de falla $\mathrm{F}$, respectivamente.
Para el caso de una falla monofásica entre la fase A y tierra, la impedancia en el nodo $F$ presentada en (10) toma la forma mostrada en (11).

Si la falla es de tipo bifásica (fase a fase o fase a tierra), por ejemplo entre A-B y tierra, (10) toma la forma presentada en (12). Igualmente ocurre para el resto de fallas entre fases o doble fase a tierra.

En caso de una falla trifásica, se usa igualmente (12), para dos de las tres fases en falla.

El cálculo de la reactancia de falla $X_{F}(m)$, en términos de la distancia $m$, se presenta en (13).

La distancia de falla en $p . u$ se encuentra para el valor de m donde $X_{F}$ adquiere el mínimo valor.

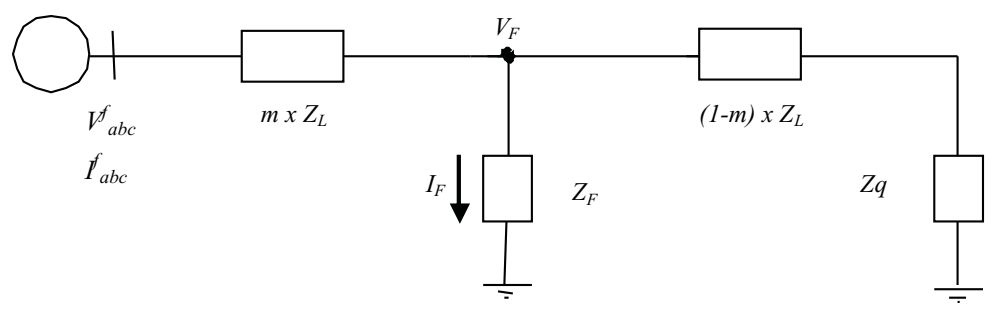

Figura 4. Circuito de distribución simplificado, ante una falla en el punto $m$

$$
\begin{aligned}
& {\left[\begin{array}{l}
V a_{F}(m) \\
V b_{F}(m) \\
V c_{F}(m)
\end{array}\right]=\left[\begin{array}{l}
V a_{f} \\
V b_{f} \\
V c_{f}
\end{array}\right]-m \times\left[\begin{array}{ccc}
Z_{a a} & Z_{a b} & Z_{a c} \\
Z_{b a} & Z_{b b} & Z_{b c} \\
Z_{c a} & Z_{c b} & Z_{c c}
\end{array}\right] \times\left[\begin{array}{c}
I a_{f} \\
I b_{f} \\
I c_{f}
\end{array}\right]} \\
& {\left[\begin{array}{l}
I a_{F}(m) \\
I b_{F}(m) \\
I c_{F}(m)
\end{array}\right]=\left[\begin{array}{l}
I a_{f} \\
I b_{f} \\
I c_{f}
\end{array}\right]-\left(\begin{array}{ccc}
\left((1-m) \times Z_{a a}\right)+Z_{q a} & (1-m) \times Z_{a b} & (1-m) \times Z_{a c} \\
(1-m) \times Z_{b a} & \left((1-m) \times Z_{b b}\right)+Z_{q b} & (1-m) \times Z_{b c} \\
(1-m) \times Z_{c a} & (1-m) \times Z_{c b} & \left((1-m) \times Z_{c c}\right)+Z_{q c}
\end{array}\right)^{-1} \times\left[\begin{array}{l}
V a_{F}(m) \\
V b_{F}(m) \\
V c_{F}(m)
\end{array}\right]} \\
& Z_{F}(m)=\frac{V_{F}(m)}{I_{F}(m)} \\
& Z_{F}(m)=\frac{V a_{F}(m)}{I a_{F}(m)} \\
& Z_{F}(m)=\frac{V a_{F}(m)-V b_{F}(m)}{I a_{F}(m)-I b_{F}(m)} \\
& X_{F}(m)=\left|\operatorname{imag}\left(Z_{F}(m)\right)\right|
\end{aligned}
$$




\section{iii. Determinación del tipo de falla y fases involucradas}

Para determinar el tipo de falla, se utiliza el algoritmo propuesto en (Das, 1998), el cual propone que la corriente de línea en operación normal de un circuito no supera un valor umbral Im, aun cuando el circuito se encuentra operando a máxima carga. Generalmente el valor de $I m$ es obtenido para horas pico, debido a que en ese momento se espera que el circuito se encuentre operando a máxima carga. Si la corriente de línea que se registra en la subestación supera el umbral, se asume que existe una falla en esta fase.

\section{Metodología propuesta para la integración colaborativa de estrategias de localización de fallas}

Actualmente los dispositivos de protección instalados en la cabecera de los circuitos de distribución cuentan con una gran capacidad de almacenamiento de información, la cual en muchos casos no es utilizada adecuadamente. La metodología aquí propuesta está encaminada a utilizar esta información para resolver el problema de localización de fallas. El esquema metodológico de integración del método basado en los gráficos de reactancias y el análisis de la firma del sistema se muestra en la Figura 5. Esta figura contiene las etapas necesarias para localizar una falla en un sistema de distribución, a partir de las señales de tensión y corriente medidas en la subestación. Este esquema cooperativo está compuesto de tres etapas básicas que se describen a continuación:

\section{Etapa 1: Procesamiento de los registros de tensión y de corriente}

En esta etapa aprovecha las bondades de intercambio de información entre el ATP y Matlab, permitiendo obtener de forma automática las señales de tensión y corriente registradas en la subestación de distribución, ante diferentes tipos de falla en el sistema de prueba de la Figura 1, según se describe en (Pérez et al., 2010). El diagrama de la Figura 6 muestra la metodología para el procesamiento de la señal.
La generación automática de fallas en ATP consiste en generar y simular automáticamente una cantidad de copias del sistema de distribución, sometido cada vez a una falla diferente, ya sea por su localización, por su naturaleza o por la resistencia de falla. Como resultado de la generación automática de fallas y el potencial de manipulación de datos que brinda Matlab ${ }^{\circledR}$, se obtiene una base de datos que contiene las señales de tensión y corriente, en el dominio del tiempo, medidas en la subestación, con el fin de realizar los respectivos análisis de las señales resultantes de la simulación en ATP y obtener los descriptores necesarios para el desarrollo de la metodología propuesta (Mora, 2006).

Una vez creada la base de datos en Matlab, se extraen las características asociadas a cada una de las señales de tensión y corriente de fase. Cada una de las señales debe registrar al menos un ciclo de prefalla, con el objetivo de obtener los fasores de tensión y corriente de prefalla necesarios para el desarrollo del método basado en el gráfico de reactancias. La extracción de los fasores de falla se inicia un ciclo después de detectar el inicio de la falla, con el fin de eliminar los errores asociados a la respuesta transitoria del sistema de potencia.

Las señales de corriente registradas en la subestación contienen información de la actuación de los dispositivos de protección conectados a la red. De estas señales se obtienen los instantes de tiempo en que operan las protecciones y su duración, de acuerdo con lo expuesto en el numeral 2.1. Para obtener los instantes de tiempo se pueden usar herramientas matemáticas como la transformada de Fourier, la transformada de Fourier de tiempo corto o la transformada de wavelet (TW), siendo ésta última la más adecuada (Bollen\& Gu, 2006).

La TW utiliza funciones que analiza la señal tanto en el dominio del tiempo como en el de la frecuencia. La señal que es objeto de análisis se descompone en diferentes escalas con diferentes niveles de resolución, según la función Wavelet "madre"; para efectos de análisis de calidad de onda en sistemas de potencia, la función Wavelet "madre" más usada es Daubechies 4 (Vega et al., 2008). Debido a que la señal de la Figura 2 se muestrea a una frecuencia de $10 \mathrm{kHz}$, el detalle 1 


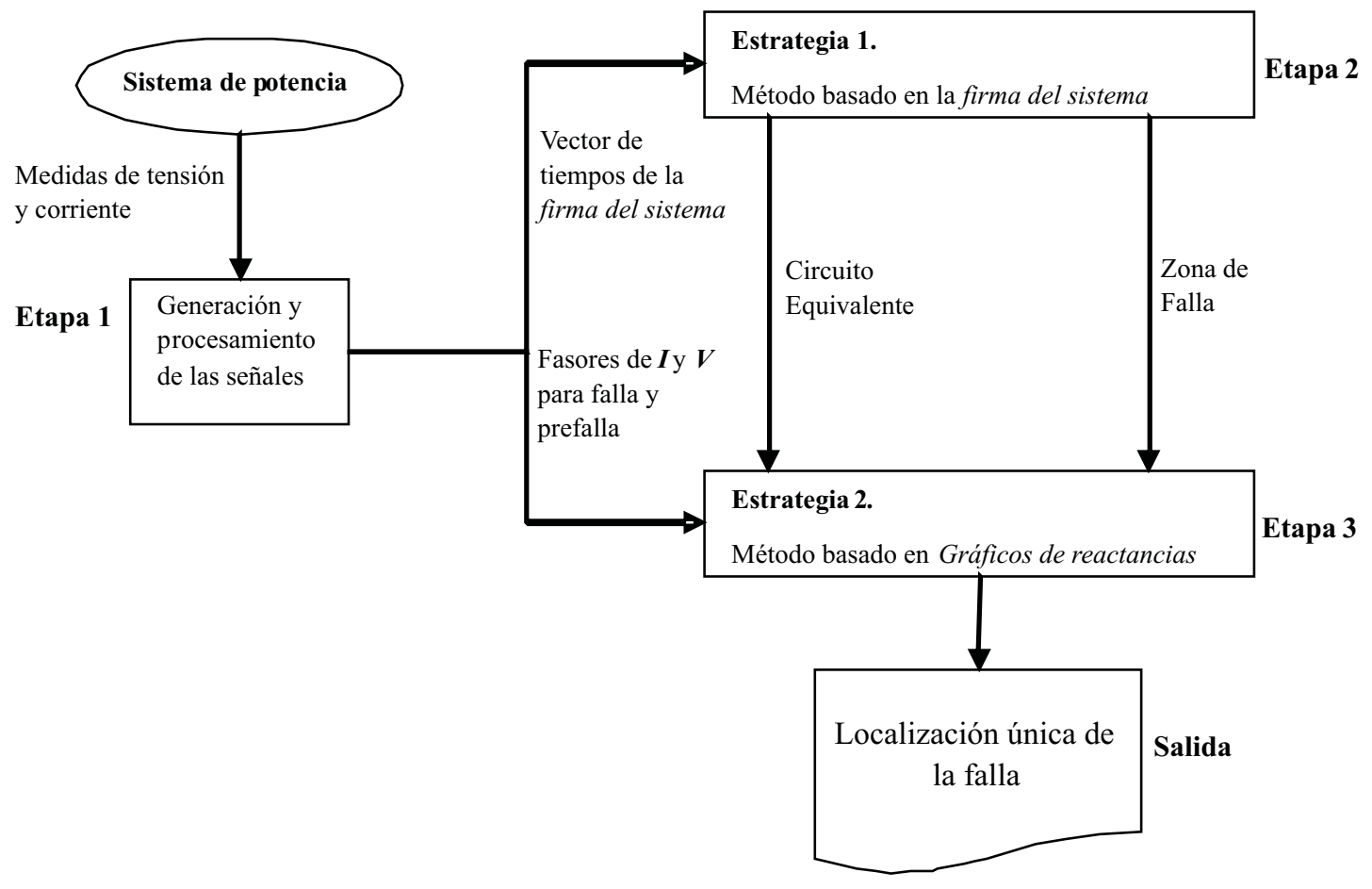

Figura 5. Metodología colaborativa propuesta para el localizador de fallas

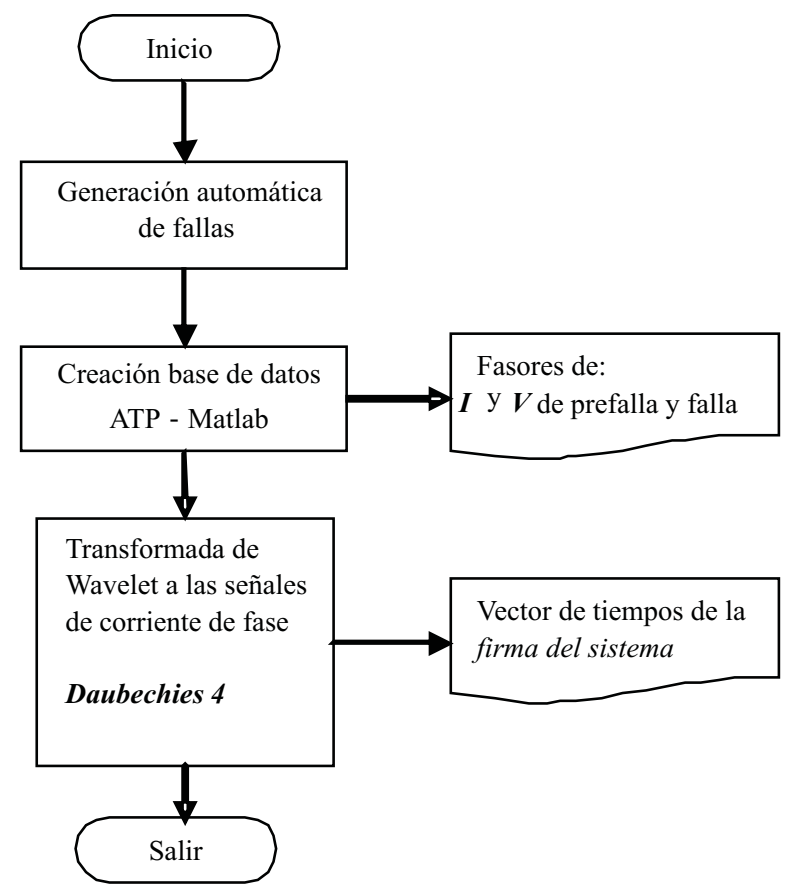

Figura 6. Metodología de procesamiento de las señales obtenidas en la subestación del circuito 
contiene frecuencias entre 2,5 y $5 \mathrm{kHz}$ aproximadamente; igualmente, el detalle 2 contiene las señales con frecuencia entre 1,25 y 2,5 kHz. La Figura 7 muestra la descomposición de la corriente en la fase B mostrada en la Figura 2, usando la transformada de Wavelet, hasta el 4 nivel de detalle.

Mediante el análisis con la TW, se pueden identificar los instantes de tiempo en que ocurren cambios significativos de la señal registrada en la subestación, que corresponden a los picos más altos de los niveles de descomposición, debido al alto nivel de armónicos presentado al conmutar un interruptor o al iniciar una falla. Una vez se identifican estos instantes, se almacenan en un vector, formando así el vector de tiempos de la firma del sistema, el cual también contiene la corriente de actuación de los dispositivos de protección.

\section{Etapa 2: Estrategia basada en la firma del} sistema.

A partir del vector de tiempos de la firma del sistema, se determinan los dispositivos que actuaron para despejar la falla y su secuencia de operación, de acuerdo con la comparación con los ajustes reales de los dispositivos instalados en el circuito. Para la señal de la Figura 2, los dispositivos identificados al despejar la falla fueron RC1 y F1. La secuencia de operación seguida para el despeje tiene inicialmente tres operaciones consecutivas de $\mathrm{RC} 1$ mediante su curva lenta, y finalmente una operación de F1, lo cual permite aislar la zona en falla. Esta secuencia de operaciones se presenta únicamente para fallas localizadas aguas abajo de F1, por tanto, a partir de la secuencia de operaciones identificada, se determina la zona en falla, según los dispositivos de protección implicados. En este caso solamente
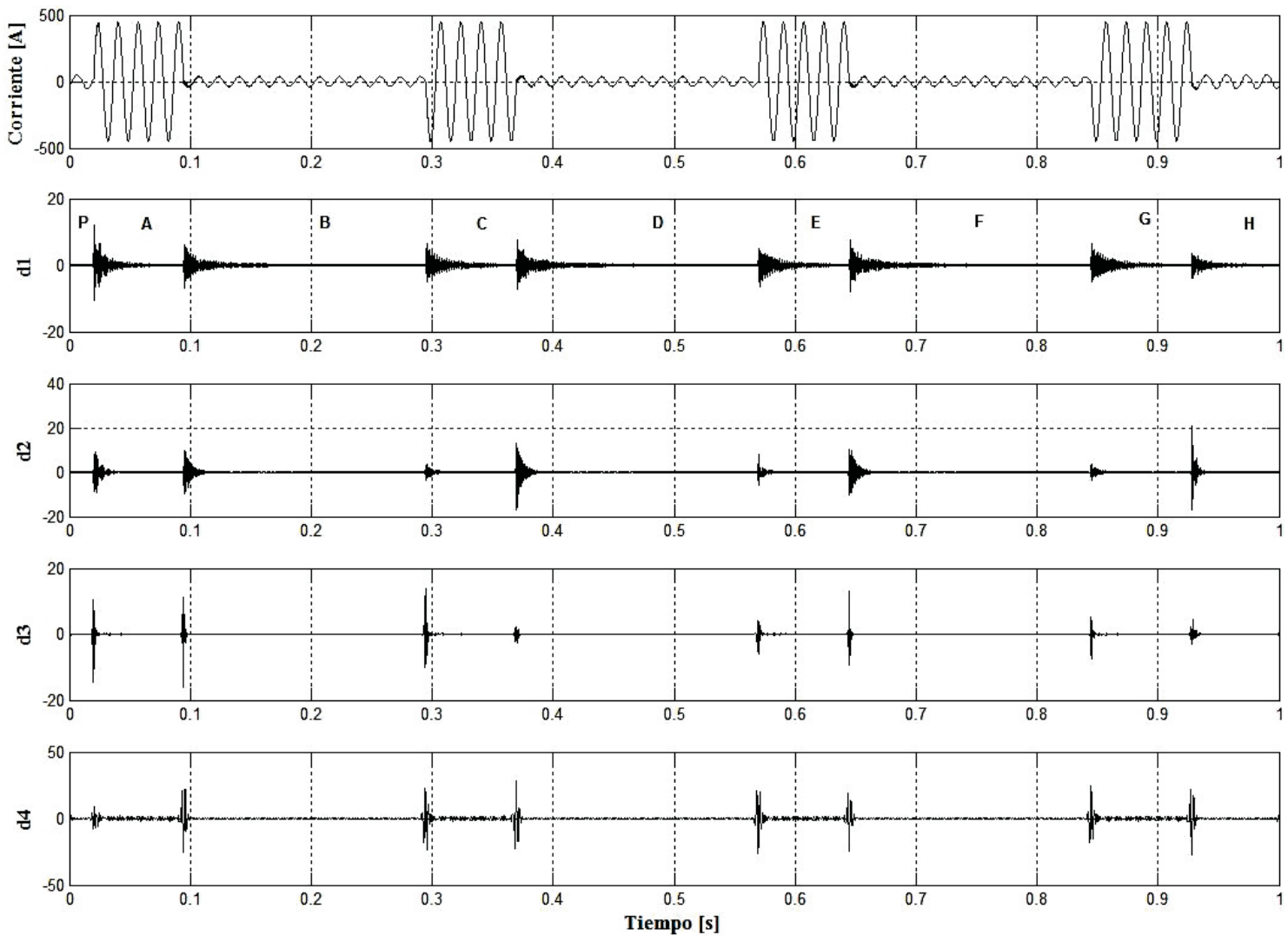

Figura 7. Detalles wavelet para la señal de la Figura 2 
es necesario zonificar el circuito de acuerdo con la zona primaria de protección de uno o un conjunto de dispositivos, para identificar de forma rápida la zona en falla, según las protecciones identificadas como activas en el proceso. El éxito de este método radica en que en un mismo circuito, los dispositivos de protección no tengan los mismos ajustes en la calibración. Algunos detalles en la calibración que permiten una diferenciación son: el número y el tiempo de los re-cierres, el número de operaciones por curvas rápidas y lentas, entre otros; según criterio del ingeniero de protecciones. Los ajustes de los dispositivos de protección tienen variaciones sutiles (del orden de milisegundos), que en aplicaciones reales, no afectan su función principal. En consecuencia con lo anterior, la aplicación de los localizadores requiere de una revisión de los ajustes de las protecciones.

Luego de identificar la zona en falla, se determina el circuito radial equivalente asociado a esta zona, el cual conserva las mismas características del circuito original, y en el cual se eliminan los ramales donde se pueda presentar la múltiple estimación. Para obtener el circuito radial equivalente se realiza la suma de la impedancia de las líneas desde la subestación, pasando por el ramal principal, hasta llegar al nodo final de la zona en falla; la matriz de impedancia resultante de esta suma, corresponde a la matriz $\boldsymbol{Z}_{L}$ necesaria para implementar la estrategia basada en el análisis del gráfico de reactancias. La Figura 8 muestra el circuito radial equivalente de falla y sin cargas, asociado a la falla realizada en el nodo 19 del sistema de prueba de la Figura 1.
La carga total del circuito se acumula en el nodo final del radial equivalente y así, lo que caracteriza el circuito equivalente de falla es la matriz de impedancia serie $\boldsymbol{Z}_{L}$, resultante de la suma de las impedancias de las líneas por donde circula la corriente de falla. Otra característica importante del circuito equivalente es que no conserva los ramales donde se pueda presentar múltiple estimación.

\section{Etapa 3. Estrategia basada en el método de análisis del gráfico de las reactancias}

La Figura 9 muestra los pasos a seguir para el desarrollo de la estrategia basada en el análisis del gráfico de las reactancias.

Para explicar la estrategia basada en el análisis del gráfico de la reactancia, se divide el diagrama de flujo de la Figura 9 en cinco etapas. En la primera etapa se acumula la carga al final del circuito radial equivalente, caracterizado por la impedancia de línea $\boldsymbol{Z}_{L}$ y los fasores de tensión y corriente de prefalla medidos en la subestación $\boldsymbol{V}_{a b c}^{p}$ y $\boldsymbol{I}_{a b c}^{p}$, respectivamente. La segunda etapa toma como datos de entrada la tensión y corriente de falla medida en la subestación $\boldsymbol{V}_{a b c}^{f}$ y $\boldsymbol{I}_{a b c}^{f}$, respectivamente. A partir de estos fasores y el valor de la carga acumulada en la etapa 1 , se determinan los vectores $\boldsymbol{V}_{a b c}(\boldsymbol{m})$ y $\boldsymbol{I}_{a b c}(\boldsymbol{m})$ para el nodo en falla F, utilizando (8) y (9), respectivamente. La etapa 3 utiliza las magnitudes de las corrientes de falla medidas en la subestación, para determinar la naturaleza de la falla, según se menciona en el numeral 2.2.3. De acuerdo con la naturaleza de la falla, en la etapa 4

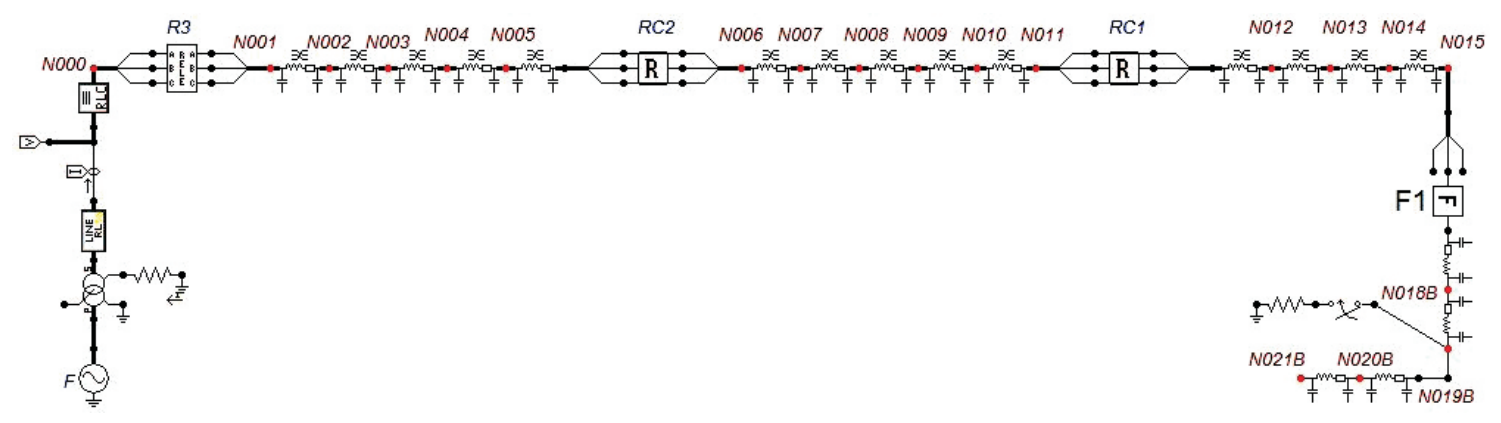

Figura 8. Circuito radial equivalente de falla, para una falla monofásica B-tierra, en el nodo 19 del sistema de prueba 


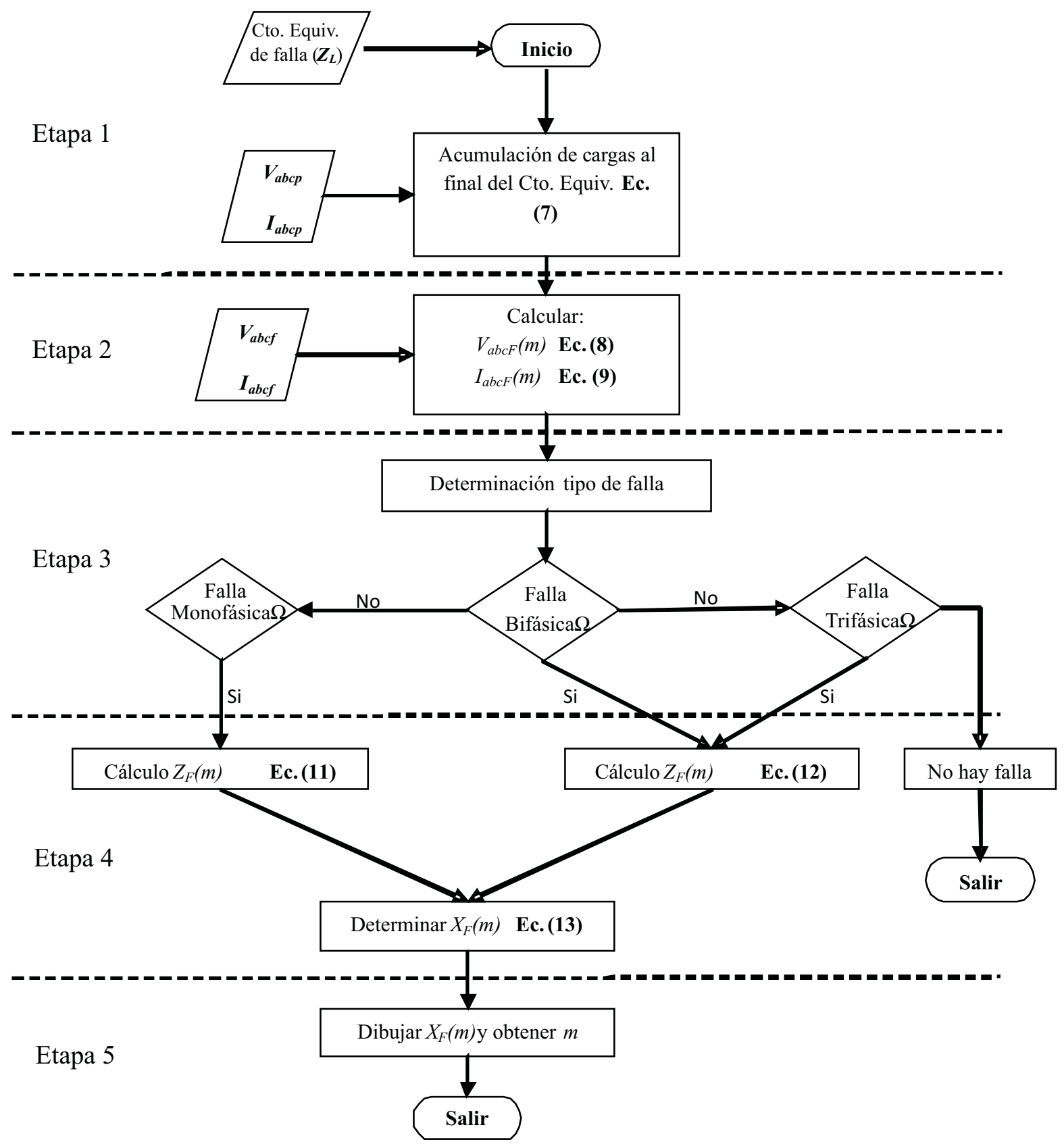

Figura 9. Diagrama de flujo para el desarrollo de la estrategia basada en el análisis del gráfico de reactancias

se calcula la reactancia de falla en función de la distancia $X_{F}(m)$. Finalmente, en la etapa 5, se estiman los valores de la reactancia de falla, variando $m$ desde $0 \mathrm{~km}$, hasta la longitud total del radial equivalente. La distancia de falla $m$ se asume que se encuentra en el punto de la gráfica donde la reactancia de falla sea mínima. Una vez se encuentra la distancia y la zona de falla, éstas se localizan en el circuito original de análisis para propósito de presentación de los resultados. 


\section{Pruebas y análisis de resultados}

\section{a. Sistema de prueba prototipo}

El sistema de prueba simplificado utilizado para mostrar la funcionalidad de la metodología propuesta se presenta en la Figura 1, modelado en el software ATPDraw y cuyos parámetros de línea se tomaron de las líneas iníciales del circuito de 34 nodos de los "test feeders" del "Distribution System Analysis Subcommitte" (Radial distribution test feeders, 1991). Para éste, R3 representa el sistema de protecciones de cabecera del circuito principal, RC1 y $\mathrm{RC} 2$ son reconectadores ubicados a $25 \mathrm{~km}$ y $11,383 \mathrm{~km}$ de la subestación, respectivamente. Finalmente, F1 y F2 son fusibles monofásicos ubicados en dos ramales del circuito. La Tabla 1 muestra los ajustes de los dispositivos de protección del circuito.

De la tabla 1, RC1 está programado para ejecutar tres operaciones por curva rápida y una por curva lenta, con un tiempo de re-cierre de $0,2 \mathrm{~s}$. RC2 realiza dos operaciones por curva rápida y una por curva lenta, con un tiempo de re-cierre de $0,05 \mathrm{~s}$.

\section{b.Zonificación del sistema de prueba}

La zonificación del sistema de prueba se muestra en la Tabla 2 y se realiza considerando la secuencia de operación de las protecciones, ante una falla permanente y suponiendo que se satisface la coordinación establecida.

En la Figura 10 se muestra el circuito de prueba debidamente zonificado, permitiendo identificar de forma rápida el sitio de ocurrencia de la falla, según las protecciones identificadas como activas en el proceso.

\section{c. Obtención de registros de fallas}

Para obtener la base de datos utilizada en la prueba de la metodología propuesta, se simularon fallas monofásicas, bifásicas y trifásicas en los nodos 3 a $13,15,16$ y 18 a 22 , pertenecientes a las diferentes zonas, con resistencias de falla de $0,5 \Omega, 20 \Omega$ y $40 \Omega$. Se debe considerar que en el sistema de prueba existen ramales monofásicos en los cuales sólo se puede simular un tipo de falla.

Tabla 1. Ajustes de los dispositivos de protección para el circuito de prueba

\begin{tabular}{cccc}
\hline Dispositivo & Curva (IEEE) & I arranque (A) & Dial \\
\hline RC1 & Moderadamente inversa & 20 & 0,07 Curva rápida - 0,1 curva lenta \\
RC2 & Moderadamente inversa & 30 & 0,34 Curva rápida - 0,5 curva lenta \\
R3 & Moderadamente inversa & 70 & 0,6 \\
F1 & Moderadamente inversa & 10 & 0,09 \\
F2 & Moderadamente inversa & 20 & 0,4 \\
\hline
\end{tabular}

Tabla 2. Zonificación del sistema de prueba, de acuerdo con la zona de falla

\begin{tabular}{cc}
\hline Secuencia de operación & Zona de falla \\
\hline RC1-RC1-RC1-F1 & Zona 1 \\
RC1-RC1-RC1-RC1 & Zona 2 \\
RC2-RC2-RC2 & Zona 3 \\
RC2-RC2-F2 & Zona 4 \\
R3 & Zona 5 \\
\hline
\end{tabular}




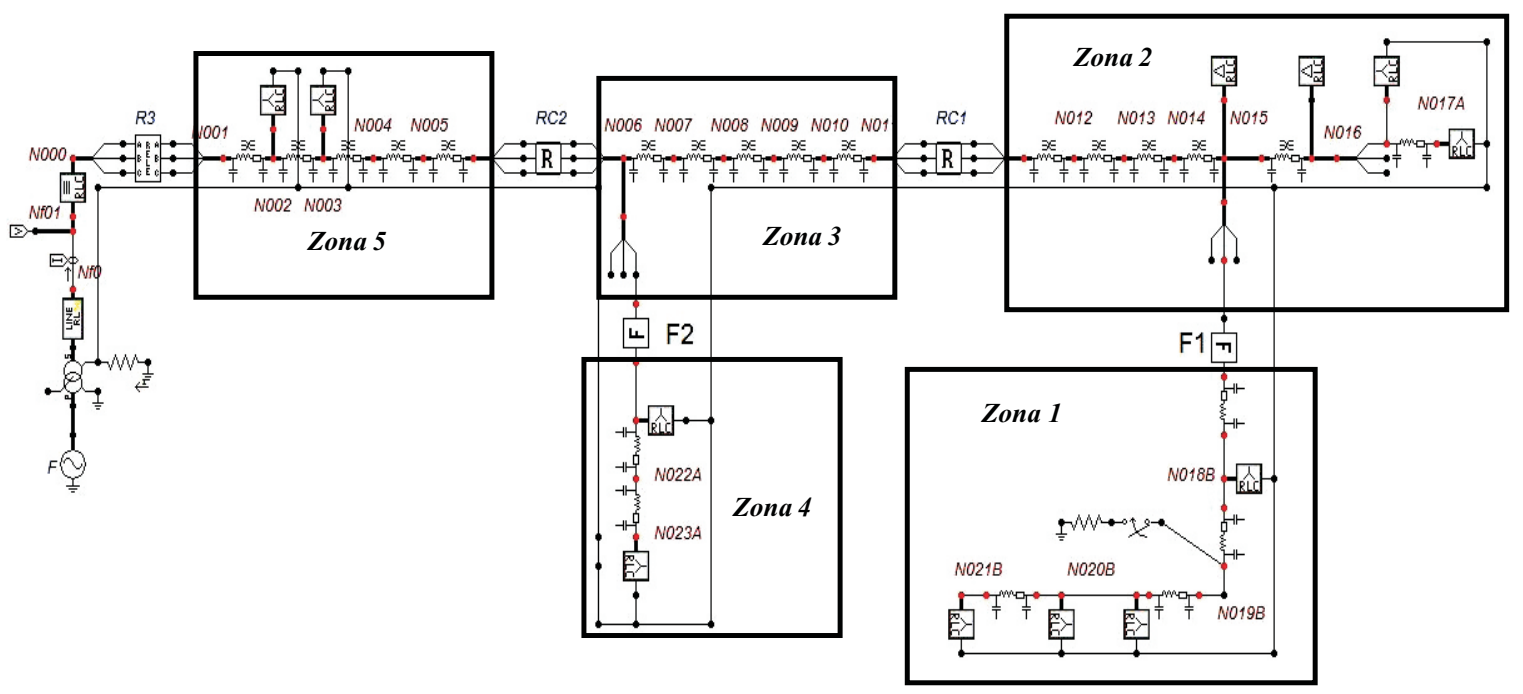

Figura 10. Sistema de pruebas zonificado

\section{d. Pruebas localizador integrado}

Las pruebas del localizador integrado comprenden diferentes tipos de fallas (monofásicas, bifásicas y trifásicas), para diferentes resistencias de falla. El error obtenido al determinar la distancia de falla se encuentra por medio de Ec.(14).

$\%$ Error $=\left|\frac{\text { Distancia }_{\text {estimada }}-\text { Distancia }_{\text {real }}}{\text { Longitud }_{\text {tramo }}}\right| \times 100$

La precisión asociada al determinar la zona de falla se encuentra por medio de Ec.(15).

Precisión $=\frac{\text { Número de registros clasificados correctamente }}{\text { Número total de registros }}$

Las Tablas 3 y 4 muestran los resultados obtenidos por el localizador integrado, para fallas de tipo monofásico, con resistencias de falla de 0,5 y 40 $\Omega$, respectivamente. Las fallas de tipo monofásico son las que registran el mayor error en la localización.

De las Tablas 3 y 4 se observa que el mayor error encontrado al determinar la distancia de falla es del 9,5\%, el cual equivale, para un tramo de 44,575 $\mathrm{km}$, a un error de $4,235 \mathrm{~km}$. Conociendo la localización de la falla, el equipo de mantenimiento puede reponerla en el menor tiempo posible, reduciendo el tiempo de permanencia del usuario sin servicio.

Para el caso de fallas bifásicas y trifásicas, los máximos errores son de 6,076\% y 1,982\%, respectivamente y ocurren para una resistencia de falla de $40 \Omega$.

La precisión en la estimación de la zona de falla, para este caso sencillo, es del 100\%, lo que resalta las bondades del análisis de las señales de corriente de falla, como una información valiosa para los métodos basados en el modelo y que permite eliminar la múltiple estimación.

Se puede decir entonces, por medio de los resultados obtenidos de las Tablas 3 y 4 y de los obtenidos para los otros tipos de falla, que el localizador integrado aquí propuesto permite la solución del problema de localización de fallas en circuitos de distribución, que utilizan elementos de protección de sobre corriente a lo largo de la red. El problema de la múltiple estimación se resuelve claramente a partir de la adecuada implementación de sus protecciones, de tal forma que además de proteger el sistema contra fallas, seccione la red por zonas. 
Ingeniería y Competitividad, Volumen 13, No. 2, p. 33 - 48 (2011)

Tabla 3. Resultados obtenidos para el localizador integrado propuesto, para fallas monofásicas con resistencia de falla de 0,5 $\Omega$

\begin{tabular}{cccccccc}
\hline \multirow{2}{*}{ Falla } & & & \multicolumn{2}{c}{ Localización real de la falla } & \multicolumn{3}{c}{ Resultado localizador integrado } \\
\cline { 3 - 8 } & R_Falla $[\Omega]$ & Nodo falla & Distancia $[\mathrm{km}]$ & Zona & Distancia $[\mathrm{km}]$ & Zona & Error $(\%)$ \\
\hline 0.5 & 3 & 1.315 & 5 & 1.275 & 5 & 0.235 \\
& 0.5 & 4 & 5 & 5 & 4.828 & 5 & 1.012 \\
& 0.5 & 5 & 10 & 5 & 9,571 & 5 & 2.523 \\
& 0.5 & 6 & 11,138 & 3 & 10,922 & 3 & 0.608 \\
& 0.5 & 7 & 15 & 3 & 14,622 & 3 & 1.063 \\
& 0.5 & 8 & 16,853 & 3 & 16,365 & 3 & 1.372 \\
& 0.5 & 9 & 20 & 3 & 19,318 & 3 & 1.919 \\
& 0.5 & 10 & 22,568 & 3 & 21,737 & 3 & 2.336 \\
& 0.5 & 11 & 25 & 3 & 23,978 & 3 & 2.872 \\
& 0.5 & 12 & 30 & 2 & 28,532 & 2 & 4.127 \\
& 0.5 & 13 & 31,630 & 2 & 29,991 & 2 & 4.609 \\
& 0.5 & 15 & 31,728 & 2 & 30,097 & 2 & 4.585 \\
& 0.5 & 16 & 33,652 & 2 & 31,876 & 2 & 4.992 \\
& 0.5 & 18 & 32,250 & 1 & 30,089 & 1 & 4.848 \\
& 0.5 & 19 & 33,576 & 1 & 31,337 & 1 & 5.023 \\
& 0.5 & 20 & 39,576 & 1 & 36,864 & 1 & 6.083 \\
0.5 & 21 & 44,575 & 1 & 41,277 & 1 & 7.399 \\
0.5 & 22 & 15 & 4 & 14,535 & 4 & 2.735 \\
\hline
\end{tabular}

Tabla 4. Resultados obtenidos para el localizador integrado propuesto, para fallas monofásicas con resistencia de falla de $40 \Omega$

\begin{tabular}{cccccccc}
\hline \multirow{2}{*}{ Falla } & & \multicolumn{2}{c}{ Localización real de la falla } & \multicolumn{3}{c}{ Resultado localizador integrado } \\
\cline { 3 - 7 } & R_Falla $[\Omega]$ & Nodo falla & Distancia $[\mathrm{km}]$ & Zona & Distancia $[\mathrm{km}]$ & Zona & Error (\%) \\
\hline 40 & 3 & 1,315 & 5 & 1,564 & 5 & 1.465 \\
& 40 & 4 & 5 & 5 & 5,049 & 5 & 0.288 \\
& 40 & 5 & 10 & 5 & 9,707 & 5 & 1.724 \\
& 40 & 6 & 11,138 & 3 & 10,957 & 3 & 0.508 \\
& 40 & 7 & 15 & 3 & 14,479 & 3 & 1.463 \\
& 40 & 8 & 16,853 & 3 & 16,187 & 3 & 1.872 \\
& 40 & 9 & 20 & 3 & 18,997 & 3 & 2.818 \\
& 40 & 10 & 22,568 & 3 & 21,274 & 3 & 3.636 \\
& 40 & 11 & 25 & 3 & 23,409 & 3 & 4.472 \\
& 40 & 12 & 30 & 2 & 27,749 & 2 & 6.327 \\
& 40 & 13 & 31,630 & 2 & 29,172 & 2 & 6.909 \\
& 40 & 13 & 31,728 & 2 & 29,243 & 2 & 6.985 \\
& 40 & 15 & 33,652 & 2 & 30,951 & 2 & 7.592 \\
& 40 & 16 & 32,250 & 1 & 29,732 & 1 & 5.648 \\
& 40 & 18 & 33,576 & 1 & 30,936 & 1 & 5.923 \\
& 40 & 19 & 39,576 & 1 & 36,151 & 1 & 7.683 \\
40 & 20 & 44,575 & 1 & 40,341 & 1 & 9.500 \\
40 & 21 & 15 & 4 & 14,416 & 4 & 3.435 \\
\hline 40 & 22 & & & & &
\end{tabular}




\section{Conclusiones}

El método híbrido propuesto integra adecuadamente el método basado en la estimación de la reactancia de falla y la firma del sistema. El primer método funciona adecuadamente bajo las condiciones propuestas de prueba, ofreciendo una alternativa de fácil implementación para la mayoría de los operadores de red, bebido a sus bajos requerimientos de información y de capacidad de cómputo. El segundo método aprovecha las diferencias sutiles en la calibración de los diferentes dispositivos que hacen parte de la automatización de la distribución, permitiendo identificar la zona en falla. Esto indica que tiene que existir una estrategia conjunta que permita ubicar y coordinar estos dispositivos con los propósitos de protección, automatización y localización de fallas.

Según los resultados obtenidos, a partir de la aplicación del localizador integrado, se puede deducir que ésta es una técnica viable a ser aplicada en sistemas de distribución que usan a lo largo de sus circuitos dispositivos de protección de sobre corriente. Únicamente se requiere de un sistema de almacenamiento de eventos, que hoy día está disponible, dado a que los dispositivos de protección de nueva generación poseen un sistema de memoria.

Una ventaja evidente que representa la aplicación de este método para las empresas distribuidoras, radica en mejoramiento de sus índices de calidad de servicio. Si se identifica el punto de falla, es más fácil para el equipo de mantenimiento de red corregir su causa, reduciendo el tiempo de permanencia del cliente sin servicio y evitando futuras fallas.

\section{Agradecimientos}

Los autores expresan su agradecimiento a Colciencias y a la Universidad Tecnológica de Pereira, en el marco del programa Jóvenes investigadores e Innovadores "Virginia Gutiérrez de Pineda".

\section{Referencias bibliográficas}

Bollen, M., \& Gu, I. (2006). Signal processing of power quality disturbances. IEEE press series on power engineering and series.

Butler, K., \& Momoh, J. (2000). A neural net based approach for fault diagnosis in distribution networks. EEE Power Engineering Society Winter Meeting, 2, 12751278.

CREG (2008). Resolución 097 de 2008. (Comisión Reguladora de Energía y Gas), Colombia.

Das, R. (1998). Determining the locations of faults in distribution systems. Doctoral Thesis, Department of Electrical Engineering. University of Saskatchewan, Saskatoon, Canada.

Gutierrez-Gallego, J., Perez-Londoño, S., \& Mora-Florez, J. (2010). Efficient adjust of a learning based fault locator for power distribution systems. IEEE/PES Transmission and Distribution Conference and Exposition: Latin America (T\&DLA), 774779.

Hagh, M., Razi, K., \& Taghizadeh, H. (2007). Fault classification and location of power transmission lines using artificial neural network. International Power Engineering Conference IPEC, 11091114.

IEEE. Radial Distribution test feeders. (1991). Distribution planning working group report. IEEE Transactions on Power Systems. 6 (3), 975-985.

IEEE Std C37.112 (1996). IEEE Standard inversetime characteristic equations for overcurrent relays. The Institute of Electrical and Electronics Engineers, Inc.

Mora, J. (2006). Localización de faltas en sistemas de distribución de energía eléctrica usando métodos basados en el modelo y métodos de clasificación basados en el conocimiento. Tesis Doctoral, Programa de doctorado en tecnologías de la información. Universitat de Girona, Girona, España. 
Mora, J., Morales, G., \& Pérez, S. (2009). Learning-based strategy for reducing the multiple estimation problem of fault zone location in radial power systems. Journal IET Generation, Transmission \& Distribution, 3 (4), 346-356.

Morales, G., Mora, J., \& Vargas, H. (2009). Elimination of multiple estimation for fault location in radial power systems by using fundamental single-end measurements. IEEE Transactions on Power Delivery, 24 (3), 1382-1389.

Morales, G., Mora, J., \& Vargas, H. (2010). Fault location method based on the determination of the minimum fault reactance for uncertainty loaded and unbalanced power distribution systems. Transmission and Distribution Conference and Exposition: Latin America (T\&D-LA), IEEE/PES, Sao Pablo, Brazil, 803-809.

Pérez, L., Pérez, S., \& Mora, J. (2010). Diseño de una herramienta eficiente de simulación automática de fallas en sistemas eléctricos de potencia. Revista Dyna, 164, 178-188.

Phadke A. \& Horowitz S. (1995). Power system relaying. Research studies press. Baldock, England.

Philipson, L., \& Lee Willis H. (2005).Understanding electric utilities and deregulations. CRC Press. 2 Edition.

Seung-Jae L., Myeon-Song C., Sang-Hee K. \& Bo-Gun J. (2004). An intelligent and efficient fault location and diagnosis scheme for radial distribution systems. IEEE Transactions on Power Delivery, 19 (2), 524-532.

Short T. (2007). Electric power distribution handbook. London, U.K. Taylor \& Francis, 1st ed.

Vega, V., Duarte, C., \& Kagan, N. (2008). Selecting the Best Wavelet Function for power quality disturbances identification patterns. 13th International Conference on Harmonics and Quality of Power IEEE. Australia. 1-6.

Zhu, J., Lubkeman, D., \& Girgis, A. (1997). Automated fault location and diagnosis on electric power distribution feeders. IEEE Transactions on Power Delivery, 12, (2), 801-809. 